\title{
FLIGHT CONTROL OF 10 GRAM INSECTS \\ BY IMPLANTED NEURAL STIMULATORS
}

Hirotaka Sato $^{1}$, Chris W. Berry ${ }^{2}$ and Michel M. Maharbiz ${ }^{1,2}$

${ }^{1}$ Electrical Engineering and Computer Science, University of California, Berkeley, CA, USA

${ }^{2}$ Electrical Engineering and Computer Science, University of Michigan, Ann Arbor, MI, USA

\begin{abstract}
We present several important advances on the flight-control microsystem for a cyborg beetle presented recently [1]. Giant beetles (Mecynorhina torquata) were employed to achieve larger payloads than the previously used Cotinis texana beetles. A new turning control method based on direct neural stimulation of the optic lobes (compound eyes) was achieved as well as initiation, cessation and elevation controls. A new variant of the initiation/cessation stimuli is also presented. Additionally, we report that operational neural stimulators and an entire working microcontroller were successfully implanted into Japanese rhinoceros beetles at the pupal stage; these beetles subsequently emerged into adults.
\end{abstract}

\section{INTRODUCTION}

Micro air vehicles (MAV's) have been the subject of intense research and development. Despite major advances, MAV's are still limited in size, payload, flight range and performance [2]. Various species of insects, among flies (Diptera), moths (Lepidoptera), dragonflies (Odonata) and beetles (Coleoptera) have unparalleled flight performance and increasingly understood muscular and nervous systems [3]. Additionally, insects undergoing complete metamorphosis (i.e. forming pupae) are very amenable to implantation and internal manipulation during pupation $[1,4,5]$. In this study, we extend our previous work to the Mecynorhina torquata, which has $7-10 \mathrm{~g}$ body weight and $1.4-2.0 \mathrm{~g}$ payload capacity.

\section{EXPERIMENTAL}

The complete microsystem is shown in Fig. 1. The neural stimulation system consisted of three neural wire electrodes: one dorsally implanted at the pupal stage between compound eyes into the insect brain and the other two were implanted either a) into the optic lobes or b) under the basalar flight muscles. A fourth wire inserted into dorsal metathorax was used as a counter electrode. As with our previous study, the neural stimulators were driven by a TI MSP430 microcontroller (some characterization used a function generator); flight command sequences were stored in the $2 \mathrm{~KB}$ memory ( $\sim 1000$ flight commands with current program). The microcontroller was powered by a microbattery which was dorsally mounted on the beetle platform. Beetles were attached on custom gimbals to measure flight responses; the torsional stiffness constant of the gimbal was $3.32 \times 10^{-5} \mathrm{Nm} / \mathrm{rad}$.

Prior to pupation, Mecynorhina makes and encases itself inside a pupal chamber. For the implantation of the neural wire electrode, first the pupal chamber was split and the electrode was inserted into the brain (Fig. 2(a)). The split pupal chambers were then glued together with a starch paste (Fig. 2(b)). For the implantation of the entire flight control system, the neural wire electrodes were soldered directly onto the microcontroller pins and all surfaces were coated with a clear nail polish. The dorsal thorax of a Japanese rhinoceros beetle was slit, and the assembly was inserted 2 weeks into the 3 week pupation period.

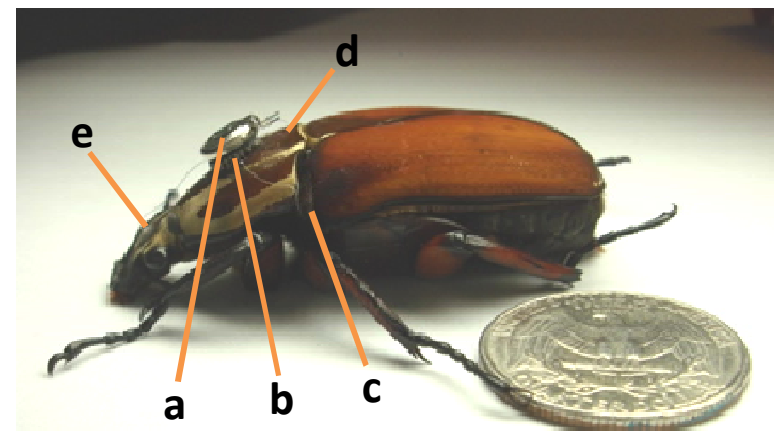

Figure 1: Cyborg beetle microsystem consisted of microbattery (a, rechargeable lithium ion coin battery, Panasonic, ML614, 3 $V, 160 \mathrm{mg}, \varnothing 6.8 \mathrm{~mm} \times 1.4 \mathrm{~mm}, 3.4 \mathrm{mAh}$ ), microcontroller (b, Texas Instruments, MSP430F2012IPWR, $63 \mathrm{mg}, 5.0 \mathrm{~mm} \times 4.5$ $\mathrm{mm} \times 1.0 \mathrm{~mm}$ ), stimulating electrodes implanted into basalar flight muscle (c), metathorax (d), brain (e), and beetle platform of Mecynorhina torquata $(7-10 \mathrm{~g}, 6-8 \mathrm{~cm})$.
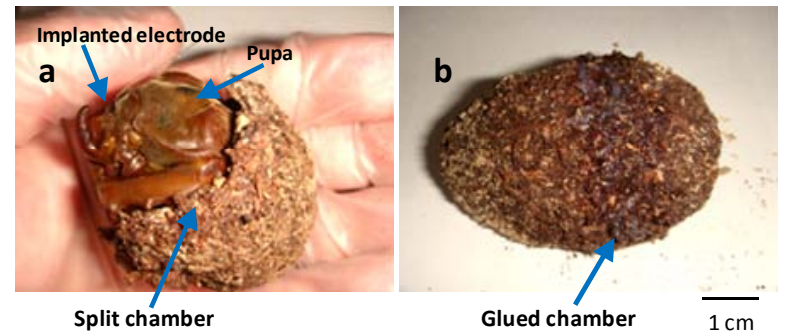

Figure 2: implantation of the neural wire electrode into a pupa of Mecynorhina torquata. (a) the electrode was inserted into the brain after splitting the pupal chamber. (b) the split chambers were glued together with a starch paste.

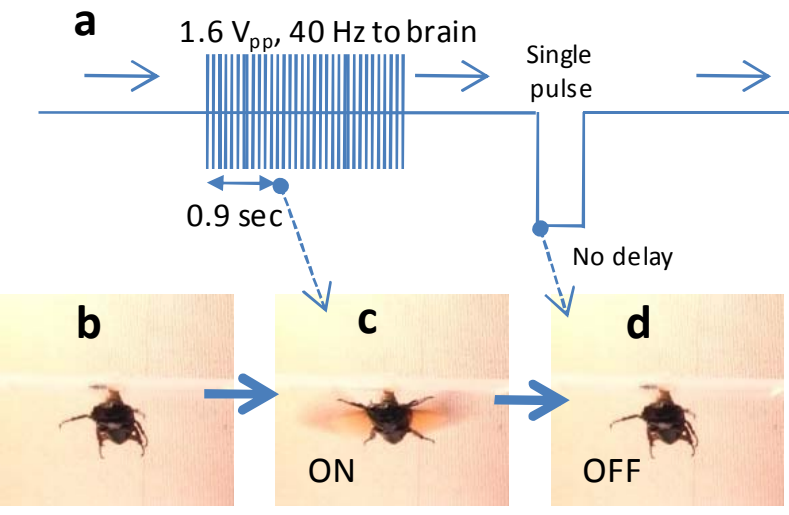

Figure 3: Initiation and cessation control of beetle flight (front views). (a) indicates typical sequential flow of pulse trains. Neural stimulation pulse trains of $1.6 V_{p p}$ and $40 \mathrm{~Hz}$ to the beetle brain started wingbeat following a $0.9 \mathrm{sec}$ induction time $(b-c)$ and $2 \mathrm{~V}$ single pulse stopped the flight without delay of response $(c-d)$. 


\section{RESULTS AND DISCUSSION}

The same on/off, throttling and turning behavior presented in [1] can be extended to other beetle systems with much larger payloads (payloads: Cotinis texana $0.2-0.4 \mathrm{~g}$, Mecynorhina torquata 1.4 - $2.0 \mathrm{~g}$, Japanese rhinoceros 1.4 - $1.8 \mathrm{~g}$ ). Figure 3 shows the initiation and cessation controls. When appropriate voltage (1.6 Vp-p on average) pulse trains at $40-110 \mathrm{~Hz}$ were applied between the right and left optic lobes, the beetle began beating wings following a short period ( $0.9 \mathrm{sec}$ on average). A single pulse of higher magnitude voltage $(>|2| \mathrm{V})$ stopped the flight without a delay of response. When initiation pulse trains were applied during flight, they lowered flight power without stopping the flight (Fig. 4). We do not yet know if both these developments (flight initiation with pulse trains and the modified throttle response) apply also to Cotinis texana. We additionally identified a new turning stimulus. Stimulation of the optic lobes with $0.8 \mathrm{~V}$ and $100 \mathrm{~Hz}$ pulse trains elicited turns towards the lobe stimulated (Fig. 5). Importantly, these results indicate that all flight controls including flight initiation, cessation, throttling and turning can be performed by using only brain-implanted neural stimulators.

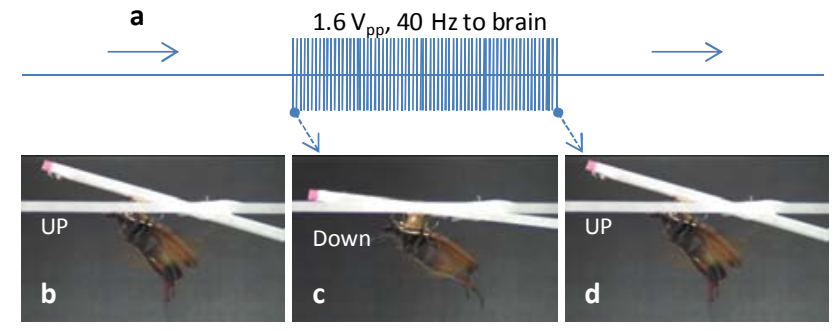

Figure 4: Elevation control of beetle flight (side views). (a) indicates typical sequential flow of pulse trains. Neural stimulation pulse trains of $1.6 V_{p p}$ and $100 \mathrm{~Hz}$ to beetle brain altered pitch of flying beetle. The beetle was mounted on a custom pitching gimbal (Fig. 2). The red marker on the gimbal follows as the beetle climed up (b, d) or down (c). The beetle decreased climbing rate whenever the stimulation pulse trains were applied to the brain. No response delay in 30 fps video was detected.
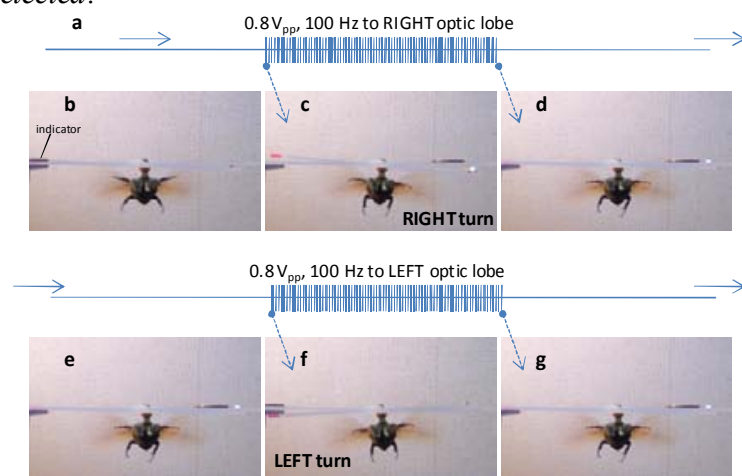

Figure 5: Tracking of optic lobe (compound eye) stimulation by pulse trains of $\sim 0.8 V_{p p}$ and $100 \mathrm{~Hz}$ (back views). The beetle was mounted on a custom rolling gimbal. (a) indicates typical sequential flow of pulse trains. The red marker on the left side of the gimbal is an indicator to signify left and right turns. The stimulation to right optic lobe elicited right turn (b-c-d), and vice versa (e-f-g). No response delay in $30 \mathrm{fps}$ video was detected.

To test the plasticity of pupal implantation, the entire system except for the microbattery was fully implanted into the dorsal metathorax of Japanese rhinocerous beetles at the pupal stage. Figure 6 shows white light and X-ray images of representative implants at both the pupal and emerged adult stages; success rate for these implants was $50 \%(\mathrm{~N}=12)$. These implanted controllers were functional in flight-ready emerged adults.
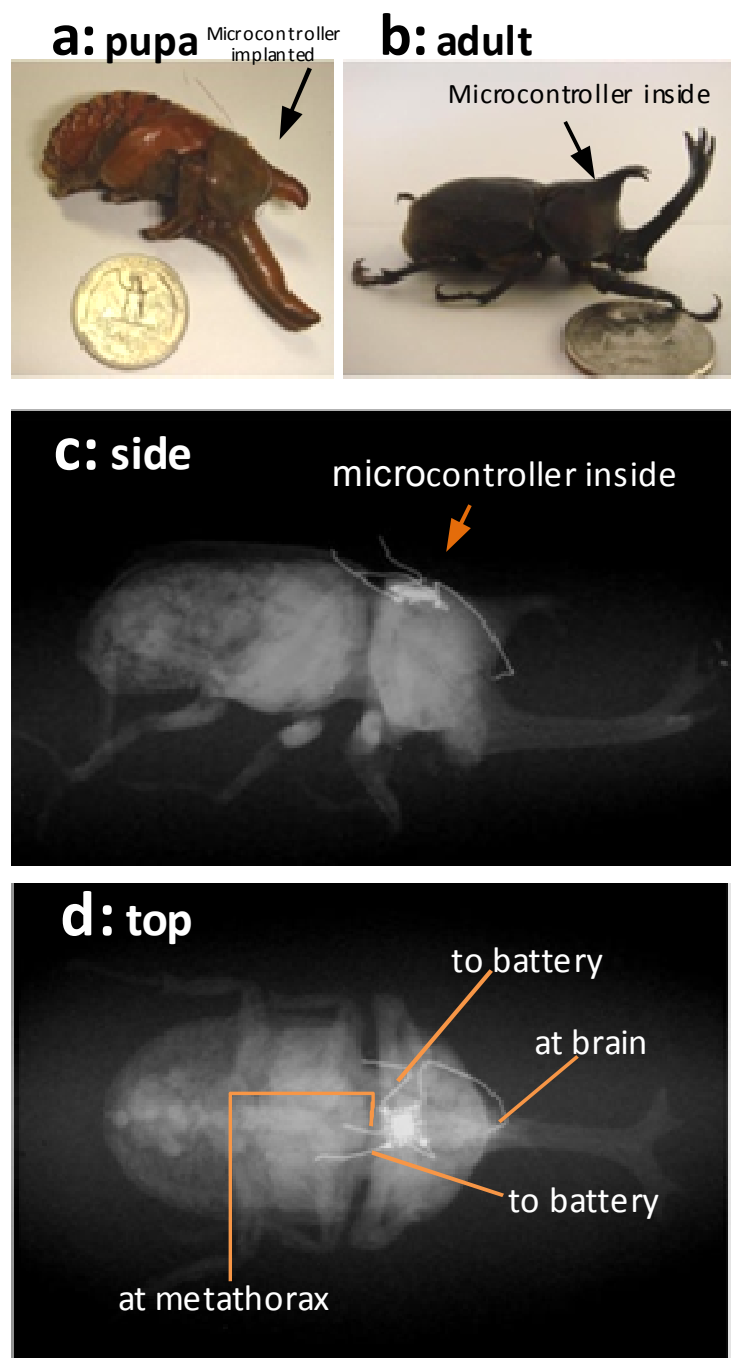

Figure 6: White light $(a, b)$ and X-ray $(c, d)$ images of entire stimulator implant; pupa (a), emerged adult (b), side and (c) top (d) views of the emerged adult. This implant was done on a Japanese rhinoceros beetle.

\section{ACKNOWLEDGEMENT}

This work was financially supported by Defense Advanced Research Projects Agency (DARPA) and Marubun Research Promotion Foundation.

\section{REFERENCES}

[1] H. Sato, C. W. Berry, B. E. Casey, G. Lavella, Y. Yao, J. M. VandenBrooks and M. M. Maharbiz, Proc. MEMS 2008, pp. 164-167.

[2] D. J. Pines and F. Bohorquez, J. Aircraft, 43 (2006), 290-305.

[3] M. Dickinson, G. Farman, M. Frye, T. Bekyarova, D. Gore, D. Maughan and T. Irving, Nature, 433 (2005), pp. 330-333.

[4]A. Bozkurt, A. Paul, S. Pulla, A. Ramkumar, B. Blossey, J. Ewer, R. Gilmour and A. Lal, Proc. MEMS 2007, pp. 405-408.

[5] A. Bozkurt, R. Gilmour, D. Stern and A. Lal, Proc. MEMS 2008, pp. 160-163. 